\title{
Analysis of Impact Force on Runner's Foot during Stance Phase
}

\author{
F. Flaviana*, R. Suryantari \\ Physics Department, Faculty of Information Technology and Science, Parahyangan Catholic University, \\ Ciumbuleuit Street No. 94, Bandung, Indonesia. \\ * Corresponding author. Tel.: +628179234003; email: flaviana@unpar.ac.id \\ Manuscript submitted November 27, 2017; accepted June 12, 2018. \\ doi: 10.17706/ijbbb.2019.9.1.51-58
}

\begin{abstract}
The aim of this research is to analyze the effect of two types of running shoes on runner's foot during stance phase, using two force plates equipped with specified track board. The main method that will be discussed in this paper is system design of gait analysis with the specific setting, in order to acquire ground reaction force (GRF) data. Then we use that experimental data to calculate the impulse during stance phase using Trapezoidal rule. The benefit of this study is to provide information and new ideas about running and its prevention over an injury.

When observed from the bar chart of the maximum GRF normalized to body weight value of the four subjects using each of the R, M, and B shoe soles, it seems that when the four subjects used footwear with $\mathrm{R}$ (Reg) sole shoe, the subject experienced the maximum force (i.e. 2.9 by the $3^{\text {rd }}$ subject) compared when using shoe sole M (Run), or barefoot B (Bare). Whereas when the subject does not use footwear (B), they did not experience a significant difference when compared with the subject of wearing footwear with shoe sole M. That also means when the $3^{\text {rd }}$ subject stands still above the force plate, it generated the force output of the gravity or the weight of the subject (the average body weight of $3^{\text {rd }}$ subject is $529.2 \mathrm{~N}$ ). The ratio of the force gain experienced by the foot of the $3^{\text {rd }}$ subject during the stance phase can reach up to 2.9 times compared to when she stood still. Total impulse that were experienced by the $4^{\text {th }}$ subject during stance phase are the summation of those two integrals (199.22 N.s and 190.52 N.s).

The measurement of foot impulse on stance phase using Trapezoidal Rule is the approximation by integrating the area under the curve in the form of polynomial function. From the impulse data that were obtained, that have not the clear correlation between sole shoes were worn with the foot impulse on stance phase.
\end{abstract}

Key words: Foot, force plate, impact force, impulse, runner.

\section{Introduction}

Many people prefer running over the other sports because it is simpler and relatively inexpensive. Running is also one of daily activities that involve physical contact between feet and ground. A runner used his lower extremities ultimately as the thrust [1].

Biomechanics is one of the interdisciplinary areas that describes, analyzes, and assesses human body motion. In Biomechanics, we learn physical activities that have the broad scope from disable's gait, weight lifting motion, until athletes performance [2]. Human often moves in his daily life without put attention to their body posture or to make efficient body movement. Here are two major applications of biomechanics, 
namely to improve human body's movement and to prevent or to treat some injuries.

Gait cycle of running is the activity that happened between one foot touched the ground and that same foot touched the ground again. One gait cycle consists of two phases, i.e. stance phase when one foot is touching the ground and swing phase when one foot is swinging or is not touching the ground [3]. Stance phase can be further divided into four stages (Fig. 1), namely:

1) Initial contact (IC)

This sub-phase is the beginning of the stance phase. One foot is about to touch the ground and the other foot does not touch the ground (or is in the swing phase).

2) Braking (B)

Shortly after one foot touches the ground, the body regulates the landing of the foot by slowing down. The knees and the heel of the foot that touch the ground will do flexion (curvature) to absorb force by the soil. During this process, the tendons and muscles store the elastic energy for the next stage.

3) Midstance (MS)

The braking phase continues until one foot is aligned right below the thigh to withstand the burden that comes from body weight. The knees and heels touching the ground are at the largest angle of flexion.

4) Propulsion (P)

When a foot that touches the ground has arranged the landing and absorbed as much energy as it could have, it will propel the body forward. The knees, heels and thighs that touch the ground do extension (straightening) to push the body up and forward using the elastic energy available during the braking phase.

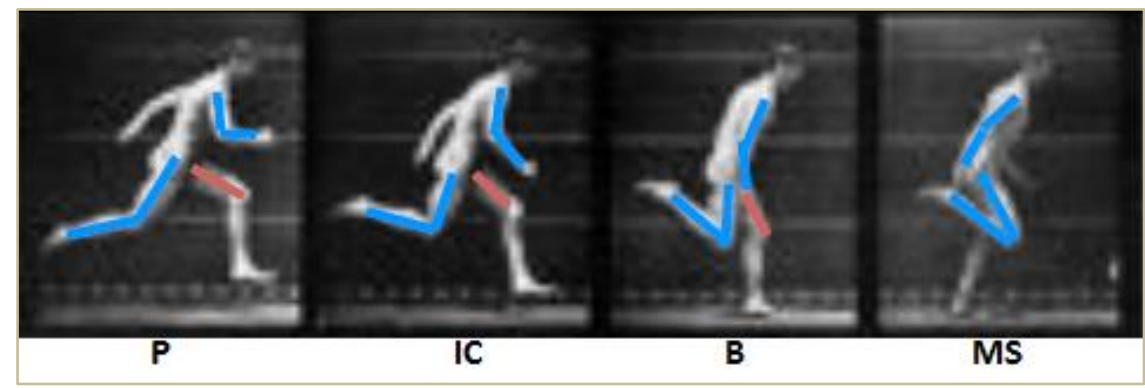

Fig. 1. Running gait cycle [3].

During running, both feet never touch the ground at the same time. Moreover, the contact time between one foot and the ground is shorter than contact time during walking. In the time of walking, one foot has contacted the ground for about $60 \%$ of one walking cycle. While in running with speed of $5 \mathrm{~m} / \mathrm{s}$, each foot has contacted the ground approximately $30 \%$ of one running cycle, and will be decreased when its velocity has been increased [4].

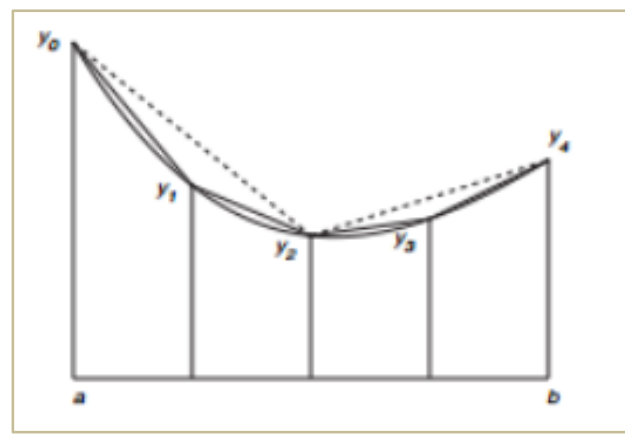

Fig. 2. Numerical integration using Trapezoidal rule [5]. 
The phases of running motion will give the information to the runners for the benefit of reaching the greatest result and body's performance. If we already have controlled the right movement pattern, all muscles, nerves, and joints will work according to the needs of running and injury rates will be decreased.

As the transition phase of the stance to the swing, one of the legs experiencing the rate of change of momentum due to contact with the ground, or so-called impulse. The impulses expressed in a graph of GRF over time, represent the area under the curve (Fig. 2). The area under a certain curve can be calculated by applying the Trapezoidal Rule [5].

\section{Methods}

GRF data were collected on Force Plate in Rooftop of $10^{\text {th }}$ building, Parahyangan Catholic University. These force plate have sampling rate about $25 \mathrm{~Hz}$, which means that both force plates calculated the forces every $0.04 \mathrm{~s}$.

GRF data of feet were taken from all the participants. The subjects were instructed to begin their running from the same initial point. We set gait analysis system to acquire the data from subjects (Fig. 3) that consists of two force plates type 0364BT with dimension $(28 \times 32 \times 5) \mathrm{cm}^{3}$ and maximum compression force $4500 \mathrm{~N}$ (named Force Plate 1 and Force Plate 2). COACH Lab II+ (Fig. 4) is the interface device that connected both force plates with Laptop. Force Plate 1 and Force Plate 2 were connected with Coach Lab II+ through British Telecom plug. The laptop was connected with Coach Lab $\mathrm{II}^{+}$through USB port. For the running track, used two wood-made boards, with dimension each $(2 \times 0.6 \times 0.05) \mathrm{m}^{3}$.

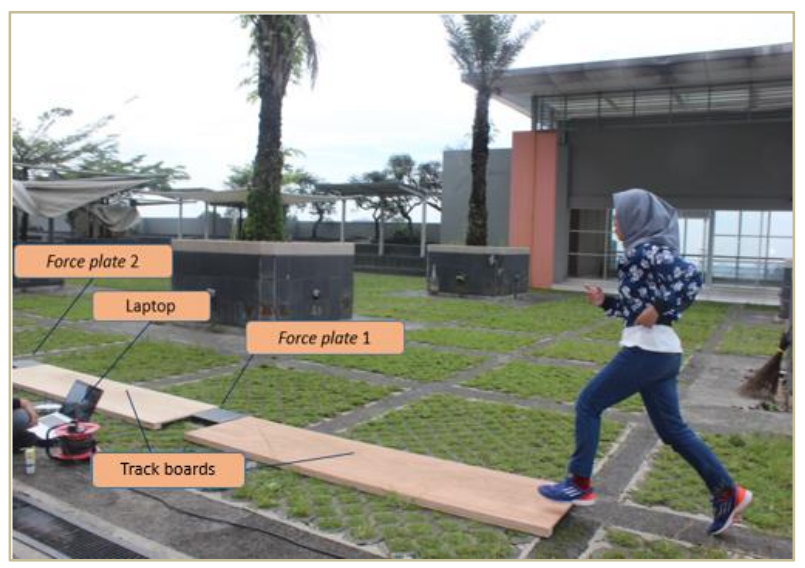

Fig. 3. Running gait system using force plate [6].

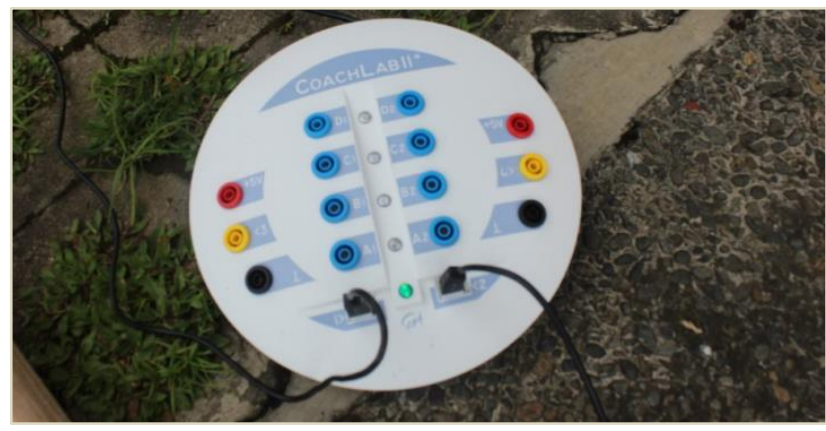

Fig. 4. Interface device COACH Lab II+ [6].

Both force plates were laid down horizontally and have the same height with the boards. They were placed separated by the boards $2 \mathrm{~m}$, Force Plate 1 was putted between the two boards, and Force Plate 2 was placed at the end of second board. The subjects were instructed to begin their running from the same 
initial point and then ran along the track. The overall running cycle took about $30 \mathrm{~s}$.

The acquired data were processed by CMA Coach Lab II that give results in form of table and graphic of GRF versus times (Fig. 5). The result data then analyzed with two techniques, using CMA software and Trapezoidal rule.

The steps of this research process were shown from Fig. 6. Four subjects are women with the same shoes size; did not have history of injuries; were recorded for 3-4 trials (rest about one minute to avoid fatique). They each ran with three kinds of shoes, i.e. Adidas Adizero running shoes with regular (R) sole shoes, Nike Air Max (M) running shoes with thick yet high flexible sole, and barefoot (B). Each subject ran 9 times, i.e. 3 sets using sole R (R1, R2, R3), 3 sets using sole M (M1, M2, M3), and 3 sets B (B1, B2, B3).

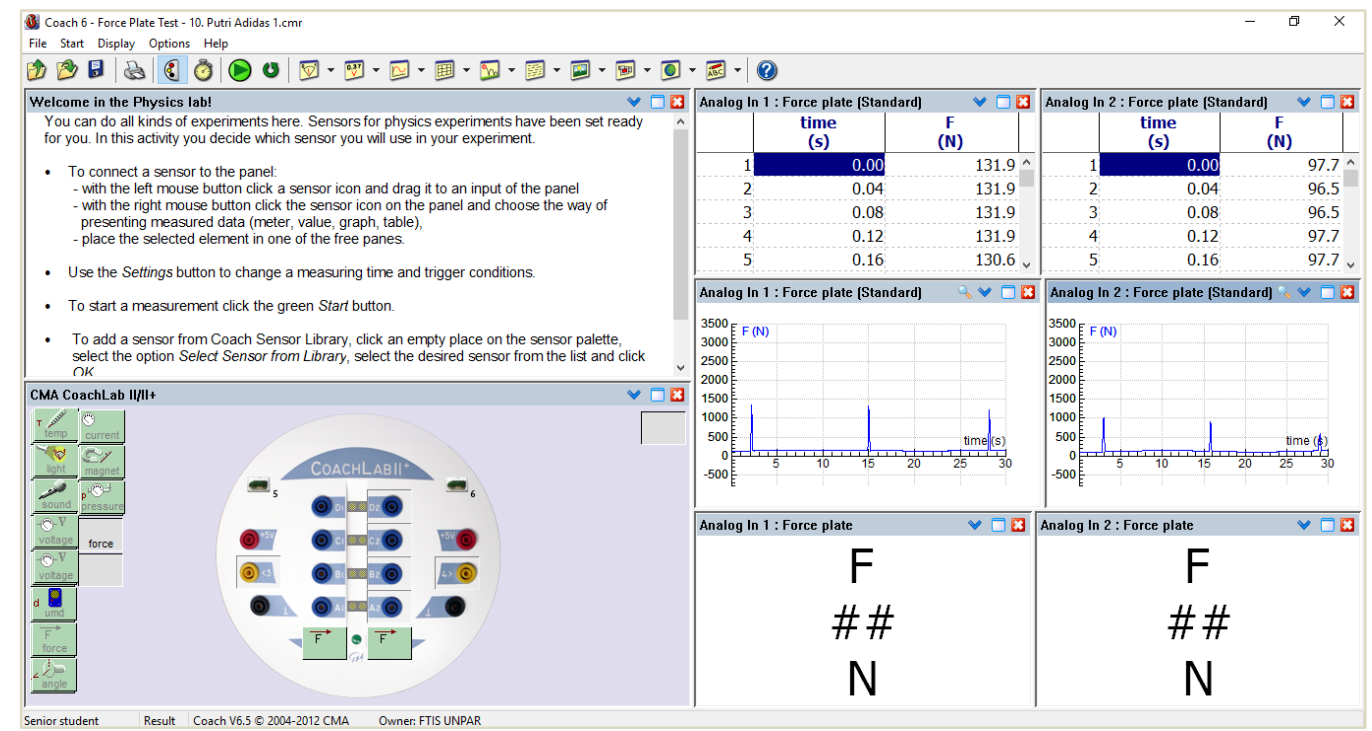

Fig. 5. Feature of CMA COACH Lab II+

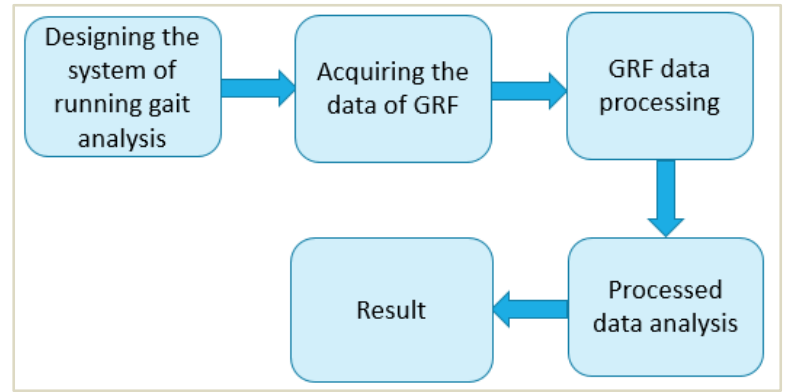

Fig. 6. The steps of research processes.

\section{Discussions and Results}

GRF data that were previously obtained have been analyzed further to calculate foot impulse during stance phase. The value of foot impulse were obtained with calculating area below the graph of GRF versus times. Fig. 7 showed the laps of running (for 30 seconds) of $3^{\text {th }}$ subject that acquired by Force Plate 1 for shoe soles R, M, and B. Moreover, information about the GRF of the same subject at the same time that acquired by Force Plate 2 are also shown in Fig. 7 .

When observed from the bar chart of the maximum GRF normalized to body weight value of the four subjects using each of the R, M, and B shoe soles (Fig. 8), it seems that when the four subjects used footwear with R (Reg) sole shoe, the subject experienced the maximum force (i.e. 2.9 by the $3^{\text {rd }}$ subject) compared 
when using shoe sole M (Run), or barefoot B (Bare). Whereas when the subject does not use footwear (B), they did not experience a significant difference when compared with the subject of wearing footwear with shoe sole $\mathrm{M}$. That also means when the $3^{\text {rd }}$ subject stands still above the force plate, it generated the force output of the gravity or the weight of the subject (the average body weight of $3^{\text {rd }}$ subject is $529.2 \mathrm{~N}$ ). The ratio of the force gain experienced by the foot of the $3^{\text {rd }}$ subject during the stance phase can reach up to 2.9 times compared to when she stood still.

Then, we set the polynom curve equation (Fig. 9-11) using Trapezoidal Rule, i.e integrating the equation upward curve GRF(t) $=-58,562.50 \mathrm{t}^{2}+538,899.50 \mathrm{t}-1,238,699.68$ with $d t$ with time boundaries $t=4.48 \mathrm{~s}$ to $t=4.6 \mathrm{~s}$. Same approximation was applied to the downward curve. Therefore, total impulse (Table 1) that were experienced by $4^{\text {th }}$ subject during stance phase (acquired by Force Plate 1 and 2) are the summation of those two integrals (199.22 N.s and 190.52 N.s).

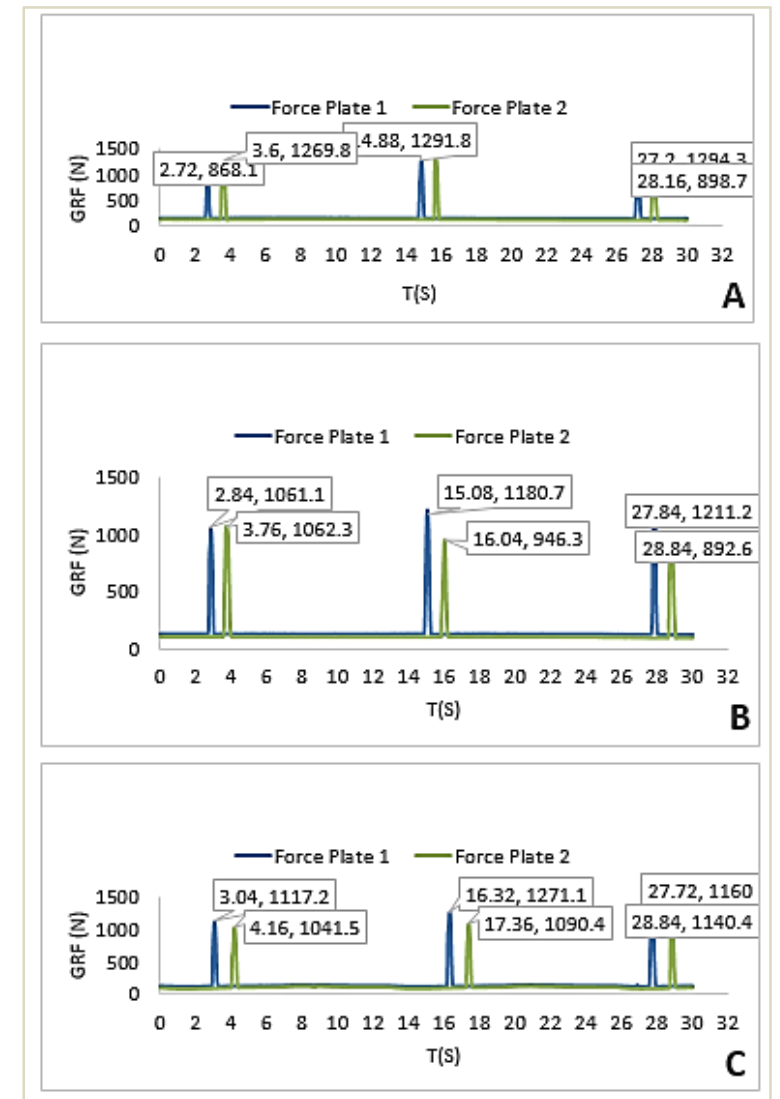

Fig. 7. The graph of GRF to time, $t$ during 30 seconds (a) set R1, (b) set M1, and (c) set B1 of $3^{\text {rd }}$ subject [6].

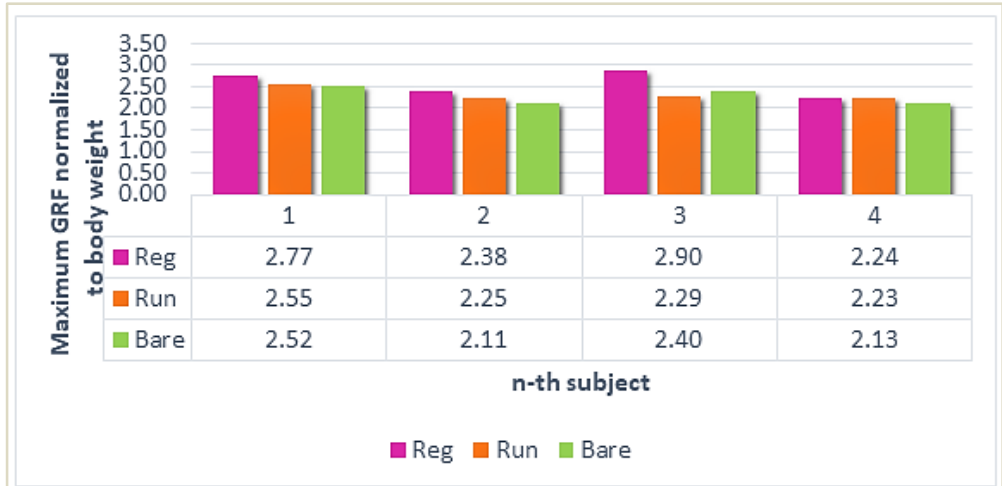

Fig. 8. The maximum GRF normalized to body weight of $\mathrm{n}^{\text {th }}$ subject for set $\mathrm{R} 1$, set $\mathrm{M} 1$, and set $\mathrm{B} 1$. 


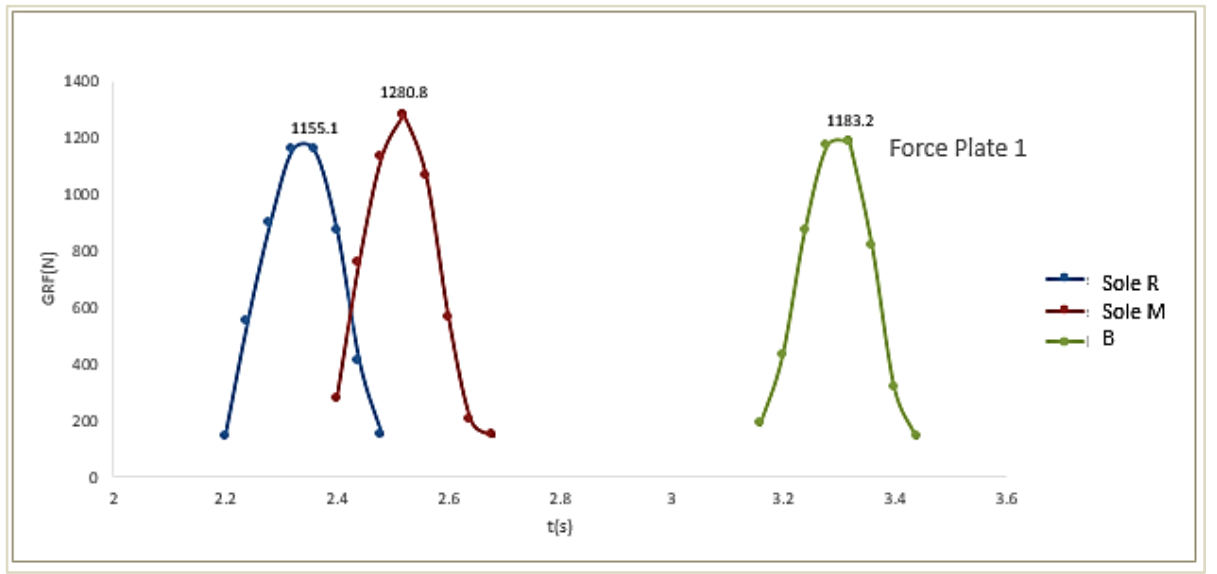

Fig. 9. The graph of GRF to time, $t$ during first lap ( $t=5 \mathrm{~s}$ ) on Force Plate 1 for R1, M1, and B1 of $4^{\text {th }}$ subject.

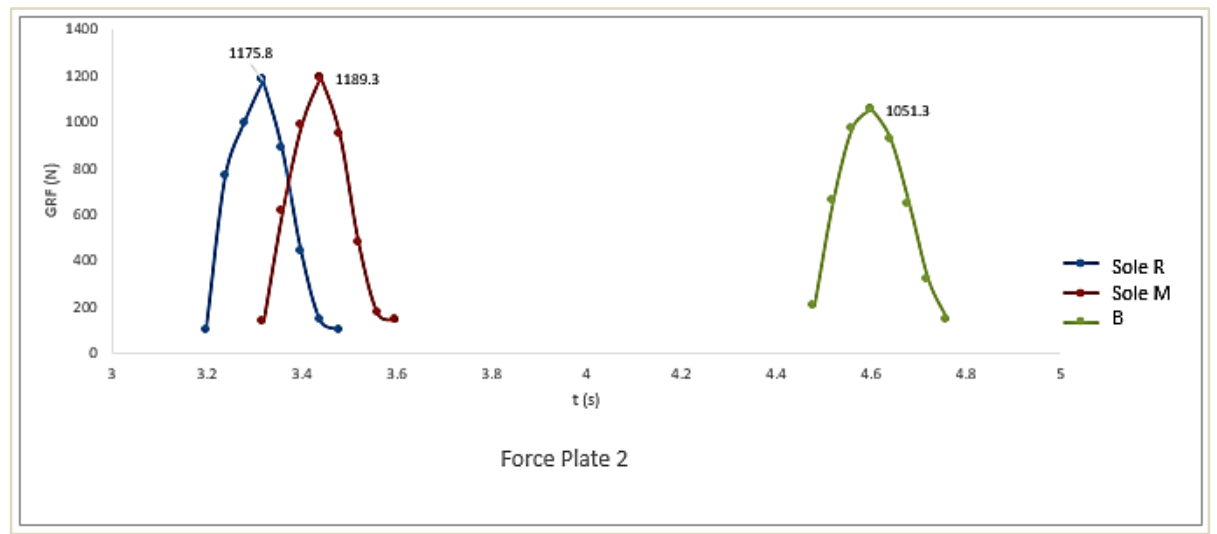

Fig. 10. The graph of GRF to time, $t$ during first lap ( $t=5 \mathrm{~s}$ ) on Force Plate 2 for R1, M1, and B1 of $4^{\text {th }}$ subject.

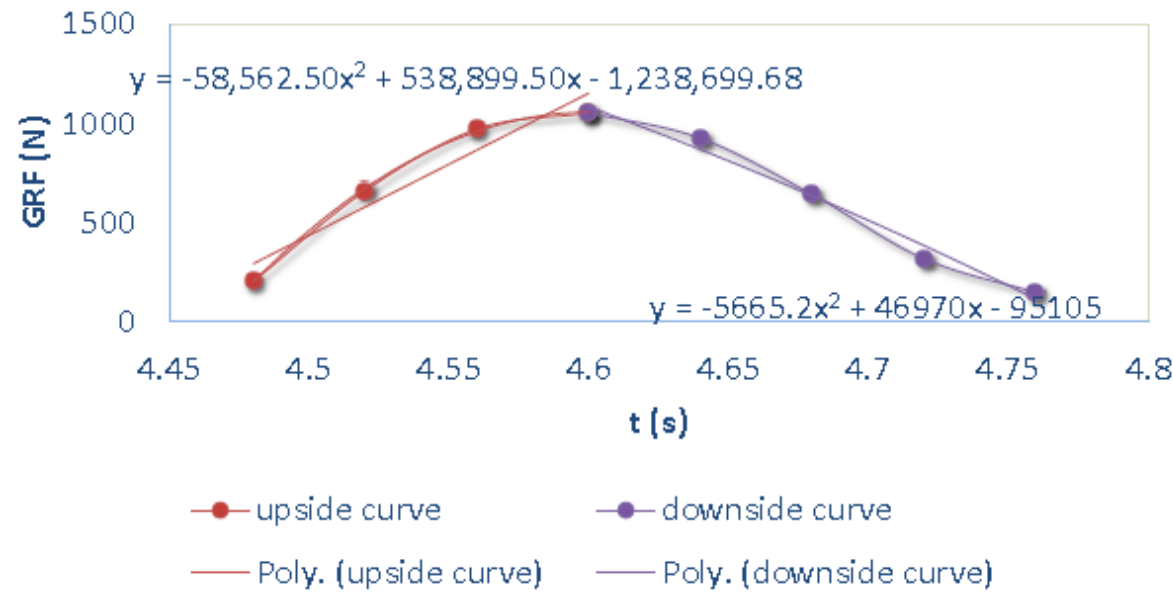

Fig. 11. The graph of GRF to times, $t$ and its polynomial equation on $4^{\text {th }}$ subject, set B, FP 2 for impulse measurement.

Table 1 showed the impulses that occur during the stance phase of each subject. The impulse value obtained varies considerably for each subject on each set of footwear soles of $\mathrm{R}, \mathrm{M}$, and $\mathrm{N}$ and there is no clear correlation between the type of sole of runner shoes with the impulses experienced. This may be due to impulse calculations performed through the Trapezoidal approach. Although the calculation of the impulse value were compared with calculating the trapezoidal area under the curve, there is indeed a difference in impulse value. 
Table 1. Impulse Data of Four Subjects at 1st Lap, Set R, M, and B

\begin{tabular}{|c|c|c|c|c|c|c|}
\hline \multirow{4}{*}{$\mathrm{n}^{\text {th }}$ subject } & \multicolumn{6}{|c|}{ Stance phase impulse (N.s) } \\
\hline & \multicolumn{2}{|c|}{ Sole R } & \multicolumn{2}{|c|}{ Sole M } & \multicolumn{2}{|c|}{ Barefoot (B) } \\
\hline & force plate 1 & & force plate 1 & & force plate 1 & \\
\hline & & force plate 2 & & force plate 2 & & force plate 2 \\
\hline 1 & 204.31 & 202.71 & 209.54 & 189.93 & 224.58 & 197.40 \\
\hline 2 & 294.02 & 289.29 & 306.87 & 270.53 & 249.71 & 263.62 \\
\hline 3 & 154.72 & 282.33 & 217.49 & 285.98 & 216.09 & 242.59 \\
\hline 4 & 160.95 & 181.10 & 208.93 & 181.81 & 199.22 & 190.52 \\
\hline
\end{tabular}

\section{Conclusions}

Subjects tend to have the highest GRF when used the regular running shoes (R), compared when running with thick sole yet high flexible running shoes (M), or barefoot (B). The magnitude of GRF that experienced by runner plays important role as the thrust of lower extremities in running, but accumulated GRFs may contribute to the risk of foot injuries, notably when using rigid sole shoes.

The measurement of foot impulse on stance phase using Trapezoidal Rule is the approximation by integrating the area under the curve in the form of polynomial function. We observed that the impulse data have not the clear correlation between sole shoes were worn with the foot impulse on stance phase.

1) In order to generate more thorough database of runner's analysis gait, any research should be conducted on specific age groups, and certain activity groups. (eg., runners and non-runners group)

2) We found another obstacle during this research, such as the lack of fund to buy certain shoes with variation number of shoes. This resulted in sample were being limited to a few subjects with relatively same shoes number.

3) In addition, dimension of the available force plate is quite small with length $\times$ width, $32 \mathrm{~cm} \times 28 \mathrm{~cm}$. Those sizes are only good for static data retrieval such as standing or jumping. When those force plates were used for data acquisition, the subjects need to adjust their running strides so that their foot falls just above each force plate.

4) Most studies just have considered the GRF. The vertical loading rate (slope of initial GRF-time curve) may be important to be observed as a prediction of how fast the GRF rises.

\section{Acknowledgment}

This work was supported by Research and Community Service Institute of Parahyangan Catholic University.

\section{References}

[1] Gross, J. M., Joseph, F., \& Elaine, R. (2009). Musculoskeletal Examination (3rd ed.). John Wiley \& Sons.

[2] Winter, D. (2009). Biomechanics and Motor Control of Human Movement (4th ed.) John Wiley and Sons.

[3] Knudson, D. (2007). Fundamentals of Biomechanics (2nd ed.) Springer.

[4] Cross, R. (1999). Standing, walking, running, and jumping on a force plate. American Association of Physics Teachers, 67(4).

[5] Dalquist, G., \& Ake, B. (2008). Numerical methods in scientific computing. Society for Industrial and Applied Mathematics, 1.

[6] Flaviana, \& Risti, S. (2016). Gait analysis study of runner using force plate. Indonesian Journal of Applied Physics, 6(2). 


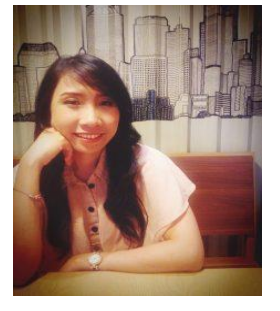

F. Flaviana was born in Jakarta, Indonesia, in October 17th, 1984. Flaviana received the S.Si. (bachelor of science) degree in physics from Parahyangan Catholic University, Bandung, Indonesia, in 2006, and the M.T. (master of engineering) degree in electrical engineering from Bandung Institute of Technology (ITB), Indonesia, in 2012.

In 2012, she joined the Department of Physics, Parahyangan Catholic University, Bandung, Indonesia, as a Lecturer until now. She has presented papers at conferences, published papers in various journals. The Characterization of Thermochromic Liquid Crystal in Temperature Distribution Measurement Using Mathematical Morphology based on the Hue Image was published in Yogyakarta: Proceedings of the $1^{\text {st }}$ International Conference on Computer Science, Electronics and Instrumentation (ICCSE) in 2012. The Application of Thermochromic Liquid Crystal in Temperature Distribution Measurement of Human's Palm Hand and Gait Analysis Study of Runner Using Force Plate were published in Surakarta: Indonesian Journal of Applied Physics, in 2015 and 2016, respectively.

Ms. Flaviana is a Member of Physical Society of Indonesia (PSI) since 2015.

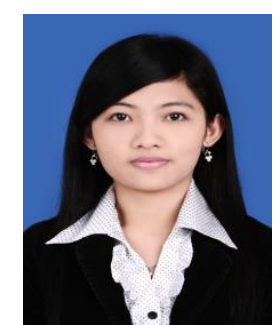

Risti Suryantari was born on 22 may 1986 in Yogyakarta, Indonesia. She works in the Department of Physics, Faculty of Information Technology and Science, Parahyangan Catholic University, Bandung, Indonesia. She was graduated from Gadjah Mada University, Indonesia in 2008, and get the master degree in 2011 at the same university. The major field of her study is the smart material as Liquid Crystal. She is active as a lecturer and researcher. 